\title{
Turbulence investigation in a Laboratory Model of the Ascending Aorta
}

\author{
1. DICEA, Sapienza Università di Roma - Via Eudossiana 18, 00184, Roma, Italy \\ 2. DICAAR, Università degli studi di Cagliari - Via Marengo 3, 09123, Cagliari, Italy
}

e-mail: stefania.fortini@uniroma1.it

\begin{abstract}
This study aims to investigate turbulence inside a model of the human ascending aorta as a function of the main flow control parameters. For this purpose, we performed a two-dimensional in vitro investigation of the pulsatile flow inside a laboratory model of a healthy aorta by varying both the Reynolds and Womersley numbers. Our findings indicate that the velocity fluctuations become significant particularly during the deceleration phase of the flow, reach the maximum near the systolic peak and then decay during the rest of the diastole phase. Higher levels of turbulence were recovered for increasing Stroke Volumes, in particular maxima of Turbulent Kinetic Energy occurred in the bulk region while higher values of Reynolds shear stresses were found in correspondence of the sinus of Valsalva.
\end{abstract}

Keywords: Ascending aorta model; Image analysis technique; Turbulence level

\section{Introduction}

Hemodynamics has become a very important research topic as the blood flow characteristics have found to play a fundamental role in cardiovascular functionality [1-3]. It is believed that both an accurate description and the assessment of blood flow features could represent a key tool to learn more about the genesis and progression of diseases. In this context, turbulence and velocity fluctuations are believed to play a role in thrombus formation, platelet activation and hemolysis [4-7]. Previous invasive in-vivo studies have assessed turbulence in the aorta both in animals [4] and in humans [8-10]. In [10] the authors observed that in healthy subjects the disturbed flow occurs in the ascending aorta and develops during the systole in correspondence of the velocity peak. Turbulent flow phenomena were observed also in the ascending aorta of subjects with aortic valvular disease, where flow disturbances were observed to occur before the systolic peak and to extend along the diastolic phase.

Medical imaging techniques have been used for clinical purposes and medical research for decades. Magnetic Resonance Imagining (MRI) has widely used to measure the bulk velocity in presence of turbulence [11] as well as to assess the Turbulent Kinetic Energy [12]. In [13] phasecontrast MRI was used to quantifying blood flow and the wall shear stresses. Recently, the 3D Cardiac Magnetic Resonance [14] has been applied to investigated the helical flows in the upper aortic arch in late systole, whereas the 4D velocity MRI
[15] has been used to test the hypothesis that age and atherosclerotic coronary artery disease (CAD) may influence aortic blood flow patterns. Finally the 4D Cardiac Magnetic Resonance (CMR) [16] allowed to characterize the relationship of aortic valve-related flow dynamics, especially with bicuspid aortic valve, and progression of ascending aortic dilation.

The Reynolds number is commonly used to define the onset of turbulent phenomena; in [8] an in-vivo critical peak Reynolds number for aortic flows has been defined. In [17] those results were improved by performing an in vitro systematic analysis of the influence of Reynolds and Womersley numbers on the development of turbulence in physiological pulsatile flows. These non-dimensional numbers are respectively defined as:

$$
\mathrm{Re}=\frac{\mathrm{UD}}{v} ; \quad \mathrm{Wo}=\sqrt{\frac{\mathrm{D}^{2}}{\mathrm{~T} v}}
$$

where $v$ is the kinematic viscosity of the blood analogue fluid, $\mathrm{U}$ and $\mathrm{D}$ are the velocity and length scales and $\mathrm{T}$ is the period of cardiac cycle.

In the past, most patient-specific computational fluid dynamics (CFD) analyses of the flow in the larger arteries, healthy aorta, aortic aneurysms, and carotid diseases considered the laminar flow assumption [18, 19] or a turbulence model was included without explicit transition modeling $[20,21]$. These studies highlighted the importance of simulating the transition to turbulence and its effects. In [22] the aortic flow was studied through nonstationary turbulence spectral analysis. The authors concluded that the temporal variation of turbulence in the aorta is rapid and that high turbulence values begin to develop close to the systolic peak, attaining their maximum in the latter half of the deceleration phase and then decaying at the end of the systole. The advent of a comprehensive transitional model [23] promised potential value for cardiovascular studies, but it needs to be tested further against a range of experimental data under physiologically relevant conditions. In this perspective, in vitro simulations allow to work in controllable and repeatable conditions. Also, the laboratory measurements performed via velocimetry techniques yield the level of accuracy required for understanding the physical phenomena, underlying then the complex structure of the flows. For more than two decades, several images analysis techniques (i.e. Particle Image Velocimetry, PIV, Particle Tracking Velocimetry, PTV, Feature Tracking, FT) have been used to perform in vitro flows measurement in cardiovascular models [24-29]. Nevertheless, despite the continuous improvements, many aspects concerning the onset of turbulence, still remain to be explored. 
In this context we investigate a two-dimensional pulsatile flow in an elastic and anatomically accurate model of a healthy aorta under a variation of the Stroke Volume SV (i.e., the volume ejected from the ventricle in one heart beat), and consequently of the Reynolds and Womersley numbers. The main aim is to investigate the turbulence in a system characterized by strong time variability, also describing the flow pattern evolution during the filling and emptying of the ascending aorta. As discussed in previous papers [28, 29], the turbulence statistics have been quantified by using a frame invariant formulation, i.e. which does not depend on the orientation of the reference frame on the measuring plane [30]. Finally, the time evolution of integral quantities, obtained from the time-resolved velocity measurements, is used to globally describe turbulence in the different experiments.

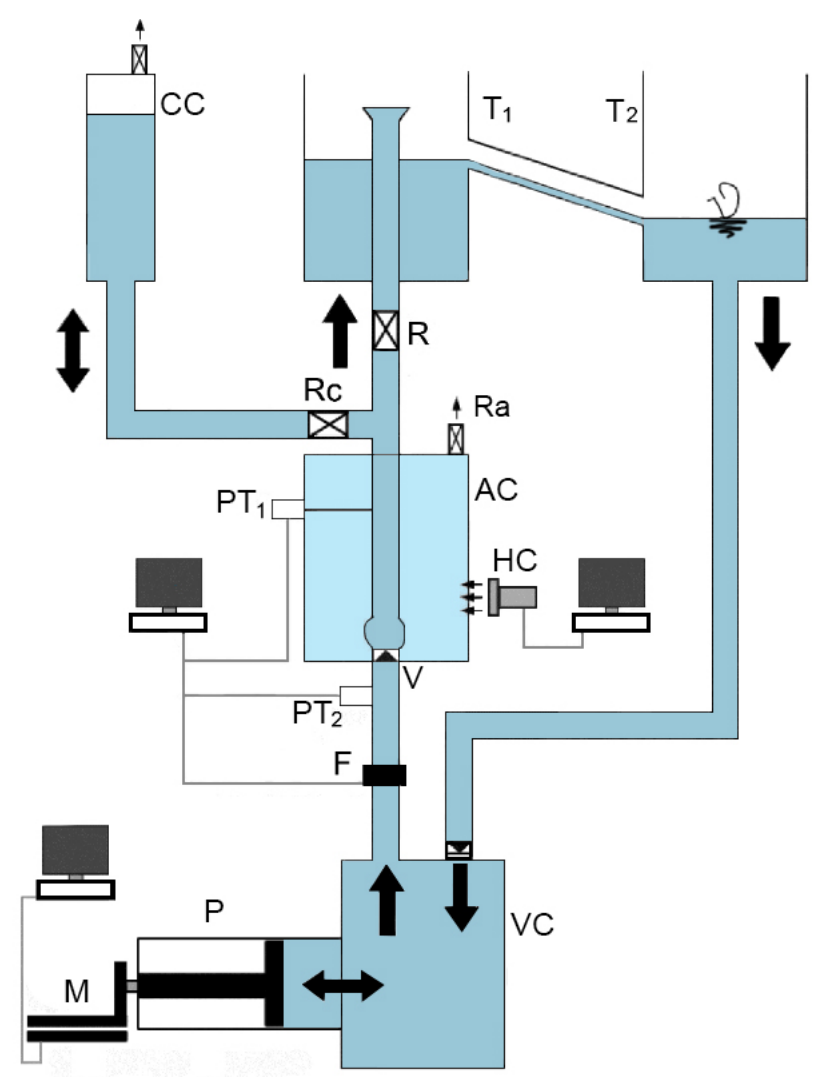

Figure 1. Experimental set up: $\mathrm{M}$ motor, $\mathrm{P}$ piston, VC Ventricle chamber, AC Aortic chamber, F flow meter, V Aortic Valve, CC compliance chamber, $\mathrm{PT}_{1}$ and $\mathrm{PT}_{2}$ pressure transducers, $\mathrm{R}$ Peripheral resistance, $\mathrm{R}_{\mathrm{C}}$ Compliance flow regulator, $\mathrm{T}_{1}$ right atrium tank, $\mathrm{T}_{2}$ left atrium tank, HC High Speed Camera, Ra resistance between Aortic chamber and atmosphere.

\section{Materials and Methods}

Figure 1 shows a scheme of the experimental apparatus. The flow inside the ascending aorta was reproduced by a hydraulic loop simulating the human systemic circulation, in both flow rate and ventricular-aortic pressure waves. A similar apparatus has been used in previous studies to investigate the flow in the left ventricle [25-29]. For this study, the anatomic district between the left ventricle outflow tract and the aortic root was accurately reproduced, also the impedance of vascular systemic net was mimicked according to a concentrated parameters approach. The pulsatile flow, was generated by a piston $(\mathrm{P})$ driven by a linear motor $(\mathrm{M})$ that is controlled by a Personal Computer. The piston displacement follows a given function governing the volume changes of the ventricle chamber (VC) in time. The aortic chamber (AC), which is the core of the apparatus, is made of plexiglass for the required optical access. The aorta model is placed inside the aortic chamber; it is made of silicon rubber (Sylgard-184, Tensile Modulus $1050 \mathrm{psi}$ and $2 \mathrm{~mm}$ thickness) to simulate both the vessel elasticity and to allow the optical access. The prosthetic aortic valve (V) used here is the pericardial tricuspid Bioprosthetic valve type 'St. Jude Medical Biocor Valve', with nominal diameter of $27 \mathrm{~mm}$, housed upstream the aortic annulus in correspondence of the sinuses of Valsalva, i.e. the three bulges at the root of the aorta, with each bulge aligned with the belly or central part of the specific valve leaflet [31].

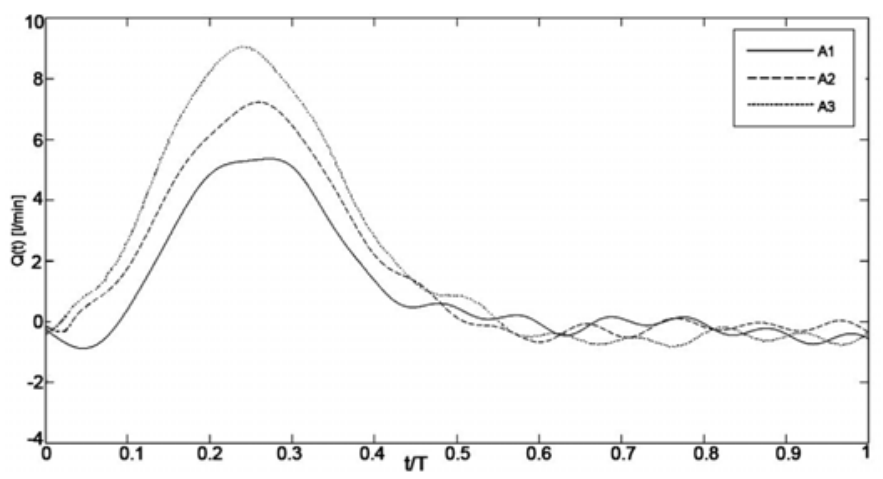

Figure 2. Flow rate recordings by the flow meter for experiments $\mathrm{A} 1, \mathrm{~A} 2$ and $\mathrm{A} 3$.

An electromagnetic flow meter $(\mathrm{F})$ is placed in order to control the real-time flow rate during the cardiac cycle; in Figure 2 the measured flow rate for all the experiments performed (see Table 1) is showed. Two piezoelectric sensors $\left(\mathrm{PT}_{1}\right.$ and $\left.\mathrm{PT}_{2}\right)$, located upstream and downstream of the valve, measure the aortic and ventricular pressures, respectively whereas the shutter tap $(\mathrm{R})$ reproduced the cardiovascular peripheral resistance. A second valve $\left(\mathrm{R}_{\mathrm{C}}\right)$ controls the flow entering or leaving the compliance chamber (CC), and an air tank that reproduces the compliance of large arteries by allowing storage/release of fluid during the simulated cardiac cycle. The aortic flow enters the right atrium tank $T_{1}$ through a nozzle open to the atmosphere, thus closing the systemic circulation. Tank $\mathrm{T}_{2}$ mimics the left atrium and feeds the VC following a pressure regime completely separated from the aortic one. The aortic root geometric aspect ratio was 1:1. For dynamic similarity to hold in this kind of flows, Reynolds and Womersley numbers should match in the physiological case and in the experimental model. The experiments were performed adopting as working fluid a solution of sodium chloride in water $(9 \mathrm{~g} / \mathrm{l}$, for the proper functioning of the flowmeter), whose viscosity was about $1 / 3$ of that of the blood. Hence, to respect the dynamic similarity, for a given physiological SV, the period of the cardiac cycle adopted in the experiments was set equal to 3 times the physiologic one.

\begin{tabular}{|c|c|c|c|c|c|}
\hline$\#$ & $\mathrm{SV}[\mathrm{ml}]$ & $\mathrm{T}[\mathrm{s}]$ & $\mathrm{U}[\mathrm{m} / \mathrm{s}]$ & $\mathrm{Re}$ & Wo \\
\hline A1 & 54 & 2.4 & 0.090 & 2174 & 15 \\
\hline A2 & 64 & 2.4 & 0.107 & 2576 & 15 \\
\hline A3 & 80 & 2.4 & 0.134 & 3220 & 15 \\
\hline
\end{tabular}

Table 1. Main experimental parameters

Table 1 summarizes the values of the main parameters in the performed experiments: the period of the cardiac cycle was fixed to $2,4 \mathrm{~s}$ whereas three values of $\mathrm{SV}$ have been 
considered: $54 \mathrm{ml}$ (experiment A1), 64ml (experiment A2) and $80 \mathrm{ml}$ (experiment A3).

The ascending aorta kinematic behaviour, i.e. its distensibility in the interval between the systole peak and the diastole, has been reproduced by imposing a correct percentage diameter change during the cardiac cycle accordingly to the physiological range. It was measured as:

$$
D \%(t)=\left(D_{a}(t)-D_{\text {dia }}\right) / D_{\text {dia }} \times 100
$$

where $D_{a}(t)$ is the instantaneous aortic diameter and $D_{\text {dia }}$ is the the minimum diameter of the proximal aorta in the diastolic phase.

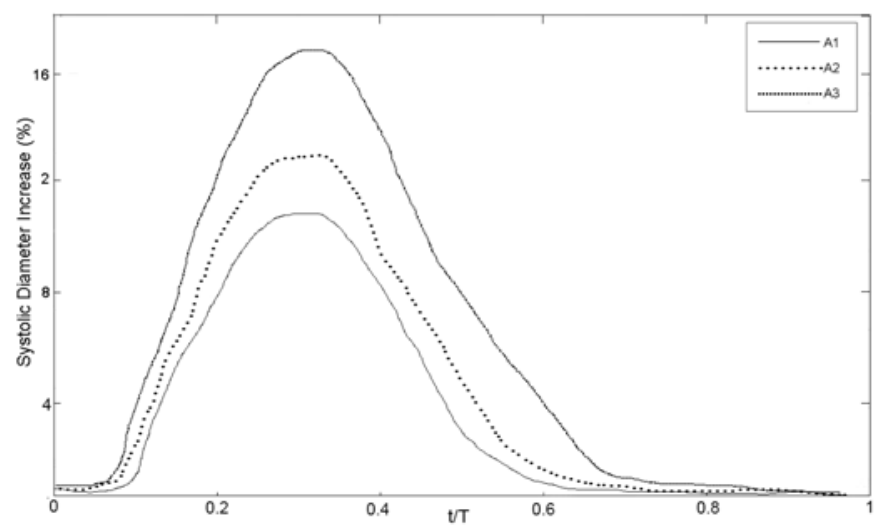

Figure 3. Percentage diameter change of the proximal ascending aorta during the cardiac cycle $\left[D \%(t)=\left(D_{a}(t)-D_{\text {dia }}\right) / D_{\text {dia }} \times 100\right]$ for all the tests performed. $\mathrm{D}_{\mathrm{a}}(\mathrm{t})$ is the instantaneous aortic diameter and $\mathrm{D}_{\text {dia }}$ is the minimum diameter of the proximal aorta. Diameters were measured by image analysis of high-speed video-recording (800 frame per second) on a section located 2.0 D downstream from the aortic valve.

In particular the resistance Ra (Figure 1) between the aortic chamber $\mathrm{AC}$ and the atmosphere was adjusted in order to match the typical values of $\Delta \%_{\max }$ identified by the in-vivo analysis of healthy subject reported in literature [32, 33]. Figure 3 shows the distensibility of the ascending aorta model during the cardiac cycle. It is worth noticing that, although most in vitro studies described in literature made use of deformable models, to the Authors' knowledge this is the first in vitro model suitable for reproducing quantitatively the aortic diameter variation during the cardiac cycle. The results obtained in terms of percentage diameter change, pressure and flow rates for all the experiments performed are summarized in Table 2.

\begin{tabular}{|c|c|c|c|c|c|}
\hline$\#$ & $\begin{array}{c}\mathrm{P}_{\text {sys }} \\
{[\mathrm{mmHg}]}\end{array}$ & $\begin{array}{c}\mathrm{P}_{\text {dias }} \\
{[\mathrm{mmHg}]}\end{array}$ & $\begin{array}{c}\mathrm{P}_{\text {mean }} \\
{[\mathrm{mmHg}]}\end{array}$ & $\begin{array}{c}\text { Diameter } \\
\text { increase } \\
\mathrm{D}[\%]\end{array}$ & $\begin{array}{c}\text { Peak } \\
\text { flow rate } \\
{[1 / \mathrm{min}]}\end{array}$ \\
\hline A1 & 113.5 & 76.2 & 87.3 & 10.7 & 5.3 \\
\hline A2 & 122.9 & 77.2 & 90.9 & 12.9 & 7.1 \\
\hline A3 & 148.6 & 80.9 & 100.5 & 17.1 & 8.9 \\
\hline
\end{tabular}

Table 2. Pressure, flow-rate and distensibility parameters

To perform velocity measurement the working fluid was seeded with neutrally buoyant particles (average diameter of $50 \mu \mathrm{m})$ and the symmetric vertical mid-plane of the aortic root (Figure 4) was illuminated with a laser light sheet $1.5 \mathrm{~mm}$ thick. Figure 4 shows the 2D plane of symmetry, on which averaged quantities involving the out-of-plane velocity component vanish. A high-speed camera (HC, 800 frames/s) with a spatial resolution $800 \times 950$ pixel acquired flow images. The considered spatio-temporal resolution $\left(\Delta \mathrm{x}_{\min }=\right.$
$0.07 \mathrm{~mm}, \Delta \mathrm{t}_{\min }=1 / 800 \mathrm{~s}$ ) was estimated suitable to identify vortex structures in the aortic root and to follow their evolution during the cardiac cycle.

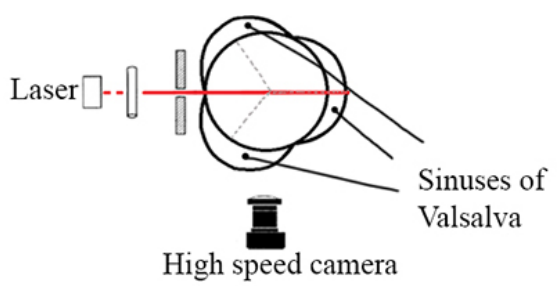

Figure 4. Sketch of the 2D measurement plane.
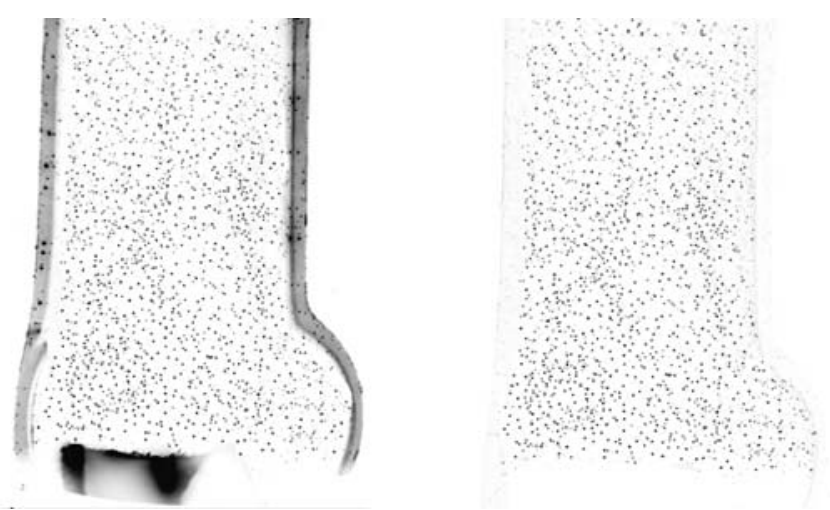

Figure 5. Row image (left) and result of the pre-processing procedure of background subtraction (right).

\section{Data analysis}

After their acquisition, images were first preprocessed in order to improve the performance of the technique and easily detect particles. In particular the procedure (Figure 5) consisted in background subtraction and thresholding segmentation on the raw images [34].

Images were then analyzed by a Feature Tracking algorithm, FT $[29,35,36]$, an image analysis technique that provides the velocity field in a Lagrangian framework. FT is a multi-frames algorithm based on the assumption that, for small time intervals, particles' light intensity is conserved between one frame and the successive, and also in both spatial directions (typically centered on seeding particles). It basically considers a measure of correlation windows between successive frames and evaluates displacements by considering the best correspondence (in terms of a defined matching measure) of selected interrogation windows between subsequent images. Lagrangian velocities are then obtained dividing the displacement by the time interval between two frames $(\sim 1 / 800$ $\mathrm{s}$ in this case). In these experiments about 6000 particles have been simultaneously traced. It is then possible to interpolate the sparse velocity vectors on a regular grid so to obtain the time sequence of the instantaneous Eulerian velocity fields. In this case the grid resolution was $50 \times 51$ : in each grid point about 25 vectors have then been interpolated using a weight function that takes into account the distance between each grid point and the point where the velocity is really measured (Figure 6). All the derived quantities needed to investigate the flow features (velocity gradients, mean flow and velocity fluctuations) can then be evaluated; it is trivial to note that the obtained flow description after the interpolation procedure cannot account for the scales smallest than the grid size. Each experimental run consisted of 100 consecutive cycles that have been used to compute phase-averaged quantities. 
Turbulent fluctuations were then evaluated considering the deviation from that average.
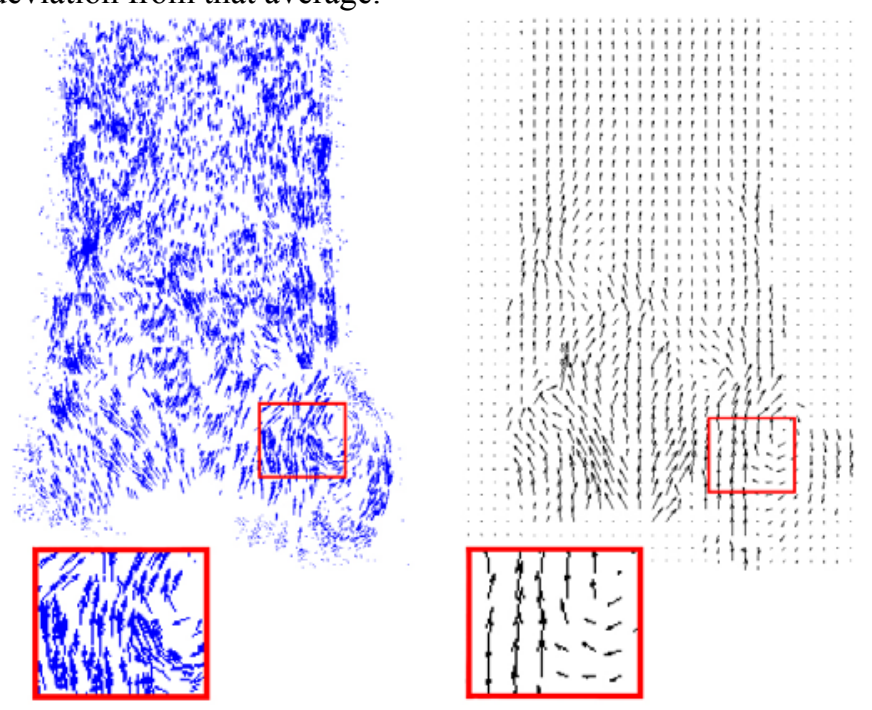

Figure 6. Instantaneous sparse velocity vectors (left) and corresponding interpolation over a regular grid (right).

\section{Results}

Turbulence was investigated using the second order moments of the velocity fluctuations. In particular, Turbulent Kinetic Energy per mass unit (TKE) and maximum Reynolds shear stress $\left(\tau_{R \max }\right)$ were considered. It has to be noted that, since $2 \mathrm{D}$ in-plane velocity measurements were performed, these quantities were evaluated from two velocity components [2931]:

$$
\mathrm{TKE}=\left(\overline{\mathrm{u}^{2}}+\overline{\mathrm{v}^{2}}\right) ; \quad \tau_{\mathrm{Rmax}}=\left(\tau_{\mathrm{R} 1}-\tau_{\mathrm{R} 2}\right) / 2
$$

where $\tau_{R 1}$ and $\tau_{R 2}$ indicate the maximum and minimum eigenvalue of the $2 D$ Reynolds stress tensor, $\tau_{R}=-\rho \overline{u_{i}^{\prime} u_{j}^{\prime}}$, i, $\mathrm{j}=1,2$.

Though symmetry arguments suggest that the maximum Reynolds shear rate is a good approximation of the real one [37], the absence of the third velocity component may affect the TKE computation. The data presented here give anyhow an insight into the onset and the intensity of turbulent phenomena. Statistics have been computed, at each fixed phase of the cycle, over the 100 cycles acquired, both TKE and $\tau_{R \max }$ have made non-dimensional by the square of the peak, spatially-averaged velocity through the aortic valve scale velocity, U. Both phase averages and time evolution of the spatial average over different regions identified in the investigated domain have been evaluated.
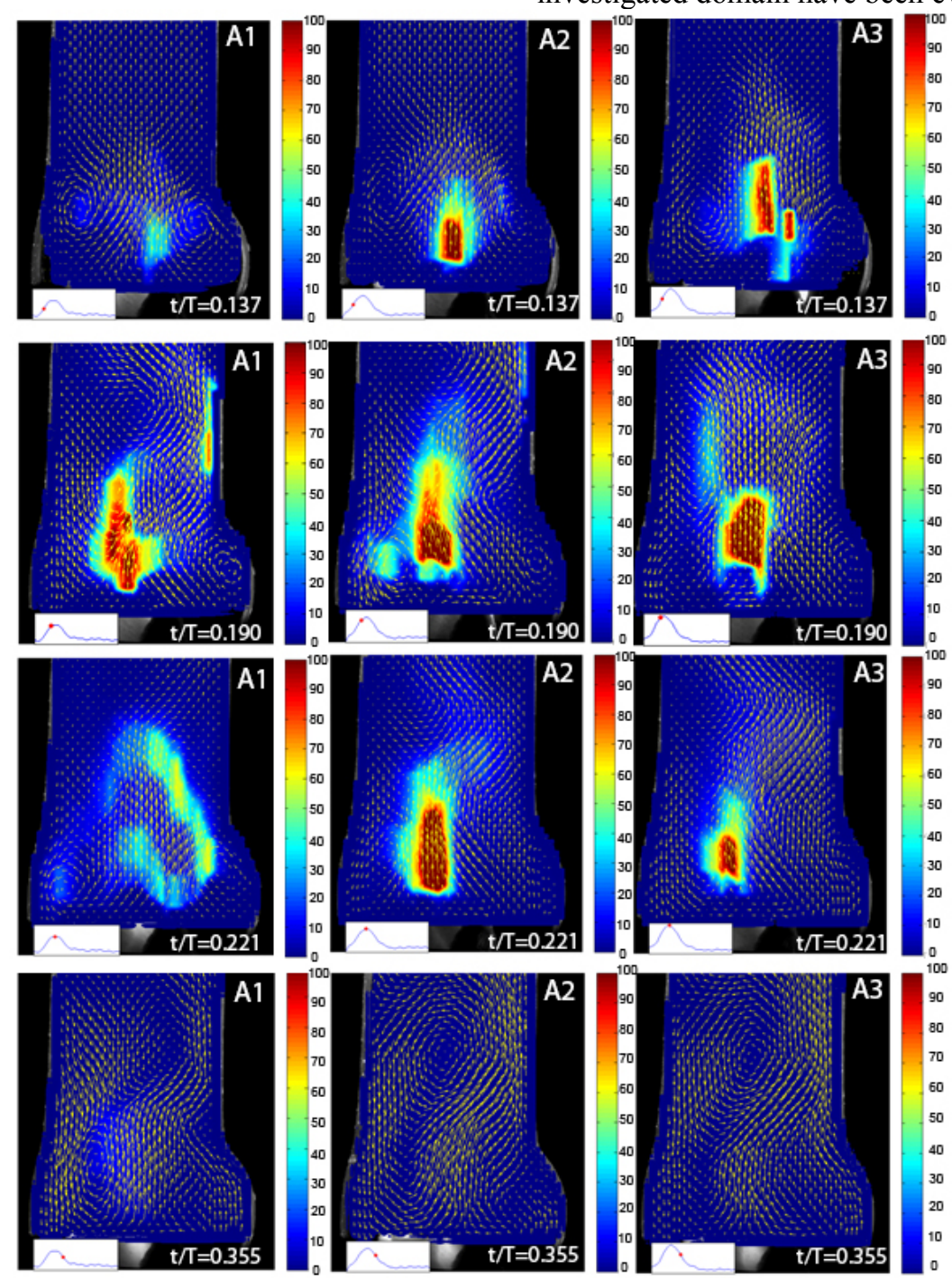

Figure 7. Instantaneous velocity field and spatial distribution of the non-dimensional TKE (colour map) correspondent to four time instants as indicated in the insert in the left corner for each plot; experiment A1 is on the left column, A2 on the central column and A3 corresponds to the right column. 


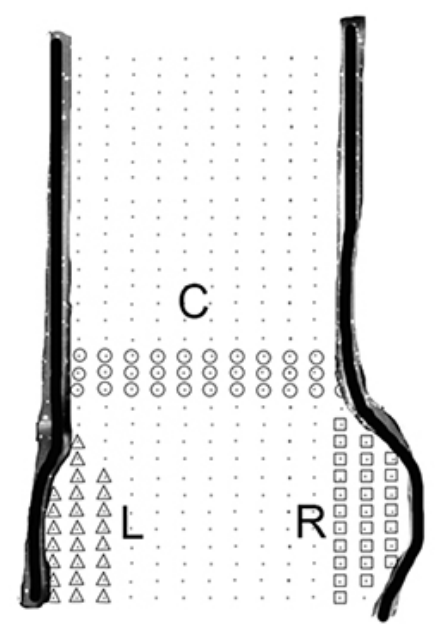

Figure 8. Different regions identified in the domain: L (left), C (centre), R (right).

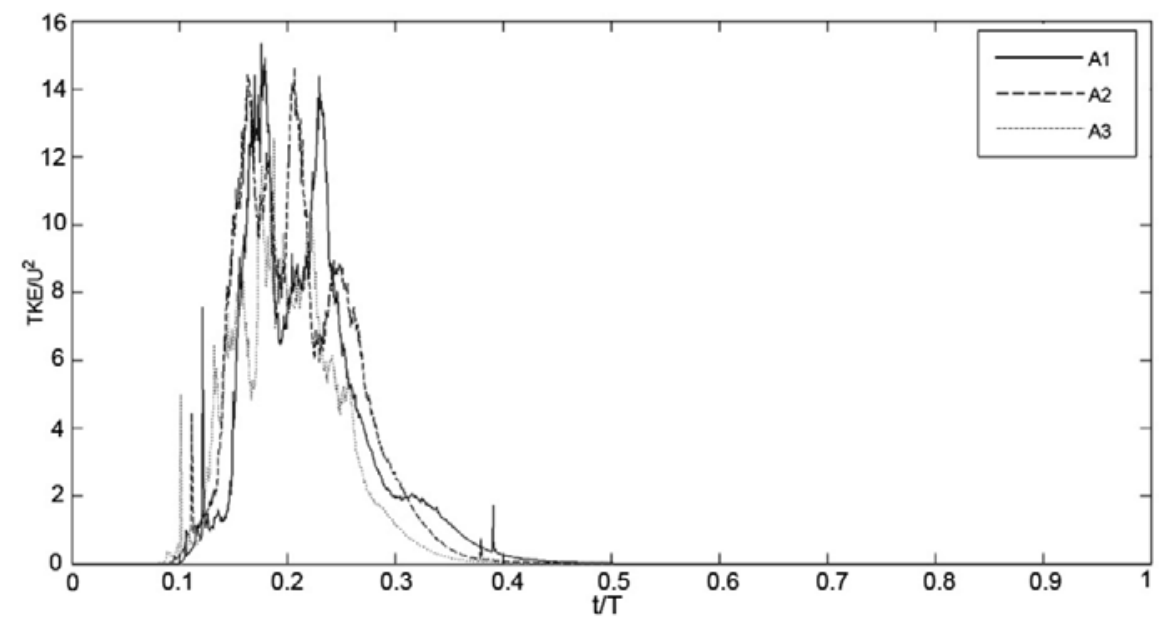

Figure 9. Non-dimensional TKE averaged over the whole domain as a function of the nondimensional time.
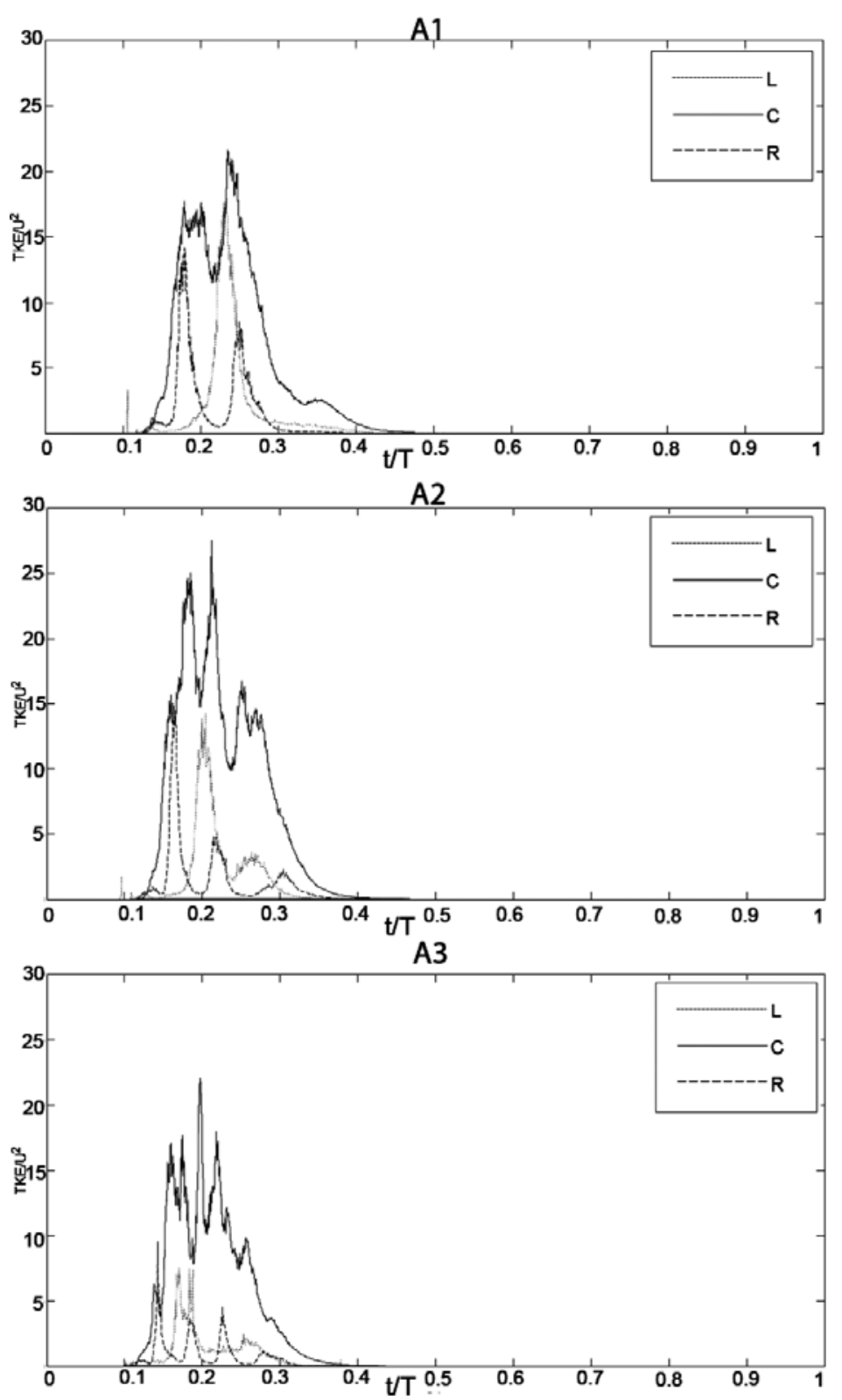

Figure 10. Non-dimensional TKE as a function of the non-dimensional time in the regions $\mathrm{L}, \mathrm{C}$ and $\mathrm{R}$ for experiments $\mathrm{A} 1, \mathrm{~A} 2$ and $\mathrm{A} 3$.

\section{Reynolds shear stresses}

Turbulence has been recognized to be among the possible causes of thrombus formation [4] as well as haemolysis [38]; as a consequence several studies [30] have been devoted to the assessment of the Reynolds shear stress level that could damage a given blood element. In [39] the time history of the stress levels experienced across the aortic root through a porcine bioprosthetic aortic valve was performed: they estimated the propensity of shear-induced damage to platelets and red blood cells.

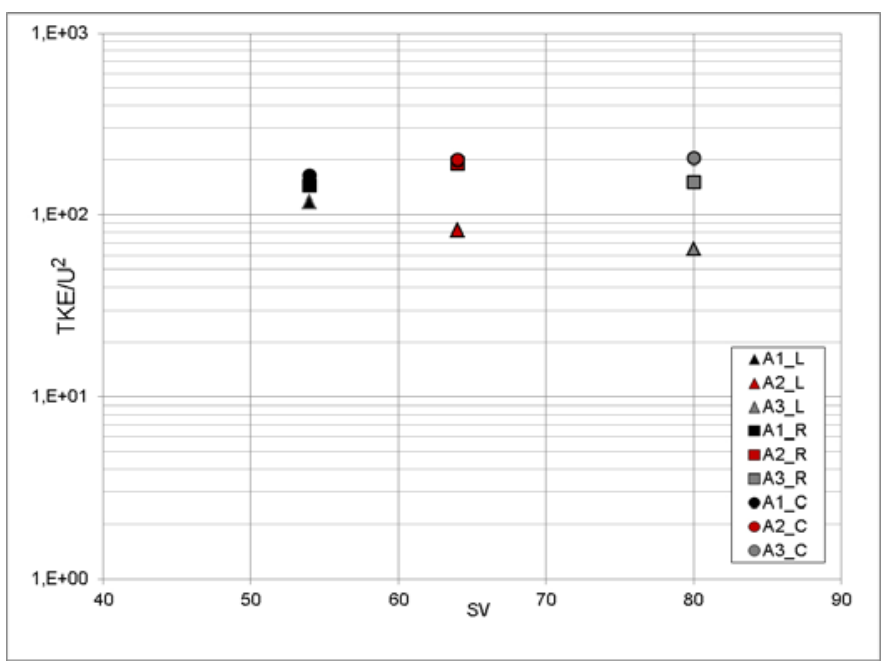

Figure 11. Maximum values of TKE recovered in the regions $\mathrm{L}, \mathrm{C}$ and $\mathrm{R}$ for all the experiments performed.

We followed the same approach proposed in [30] and estimated the maximum Reynolds shear stresses. Figure 12 shows the comparison between all the experiments of the phase averaged Reynolds shear stresses evaluated over the three different regions during the cardiac cycle. The range of values is about one order of magnitude lower than the TKE, indicating a prevalence of the normal component compared to the shear Reynolds stresses. Higher values were found in the sinus of Valsalva region (R), whereas similar values are in the commissure $(\mathrm{L})$ and in the core $(\mathrm{C})$ regions. The maximum values of Reynolds shear stresses are showed in Figure 13 in the different conditions. With the exception of the commissure 
(L), the values are similar in all the regions for all the performed experiments.
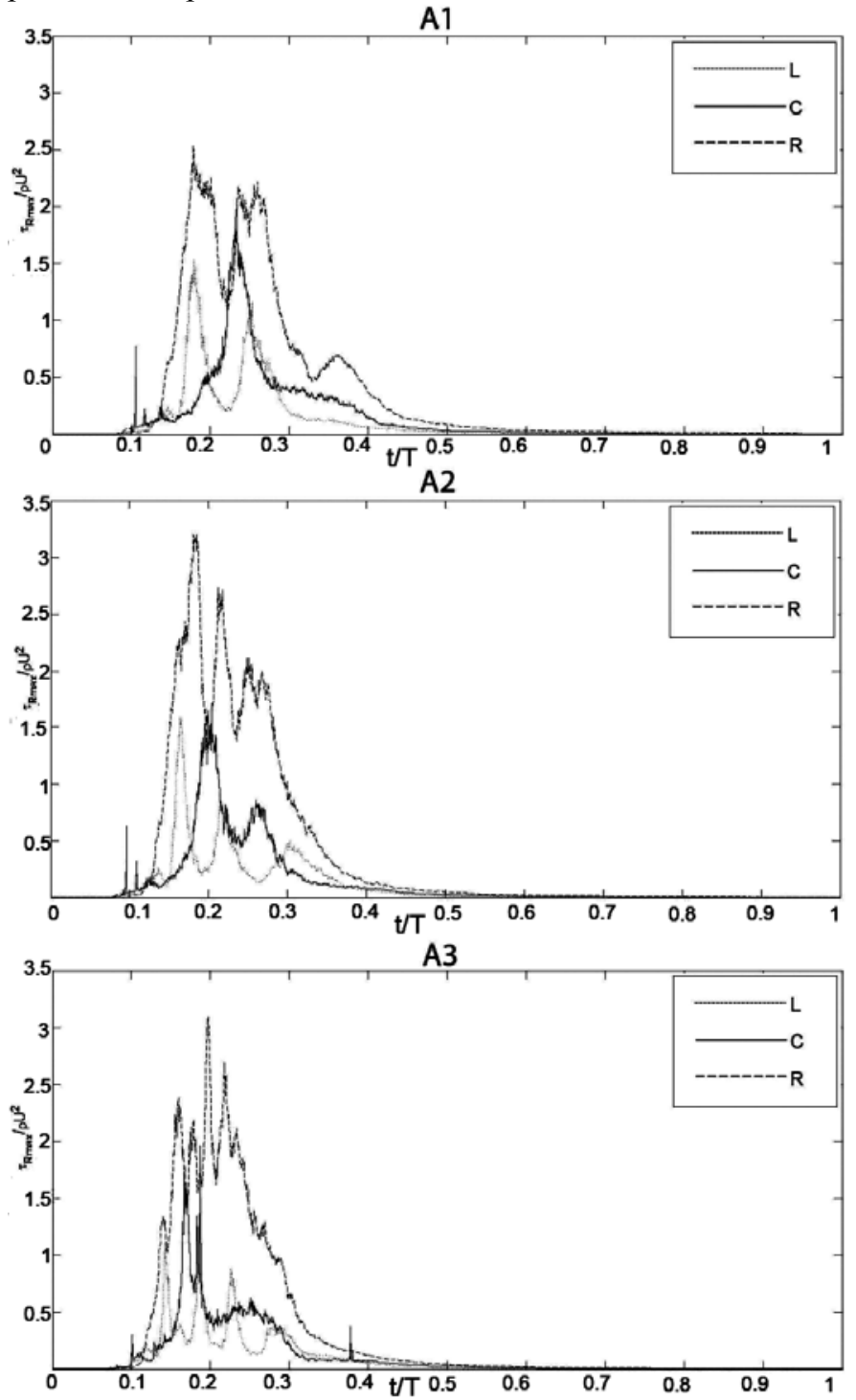

Figure 12. Non-dimensional Reynolds shear stresses as a function of the non-dimensional time in the regions $\mathrm{L}, \mathrm{C}$ and $\mathrm{R}$ for all the experiments performed.

\section{Conclusions}

An in vitro analysis of the aortic flow in a compliant model of a healthy aorta is presented. To our knowledge, the one here presented is the first model suitable to reproduce quantitatively the variation of the aortic diameter during the cardiac cycle. Experiments were carried out by varying the Stroke Volume and consequently the main control parameters, Reynolds and Womersley numbers. Each experimental run consisted of 100 consecutive cycles that were used to compute phase-averaged quantities; turbulent fluctuations were then evaluated considering the deviation from that average. Present measurements allow characterizing the flow patterns and their evolution as well as turbulent phenomena developing in the aortic root. In agreement with [8] our findings show that the turbulent kinetic energy is lower in the early systole and increases, starting from the mid systole, to reach its maximum close to the systolic peak, whereas the turbulent intensity decays during the rest of the systole. Moreover, the maximum values of TKE were recovered in the bulk region of the domain whereas the maximum of Reynolds shear stresses occurred in the sinus of Valsalva region (R).
The model of the ascending aorta was here used to investigate the physiological phenomena of the aortic filling. The same model was considered to investigate pathological situations implying a modified morphology of the aorta [40] and is currently being used to test different types of valves in both conditions. In this context, a deep investigation of the variation of the turbulence intensities and their correlation with the location and severity of cardiovascular disease may help to evaluate the role of turbulence in the development of aortic pathologies.

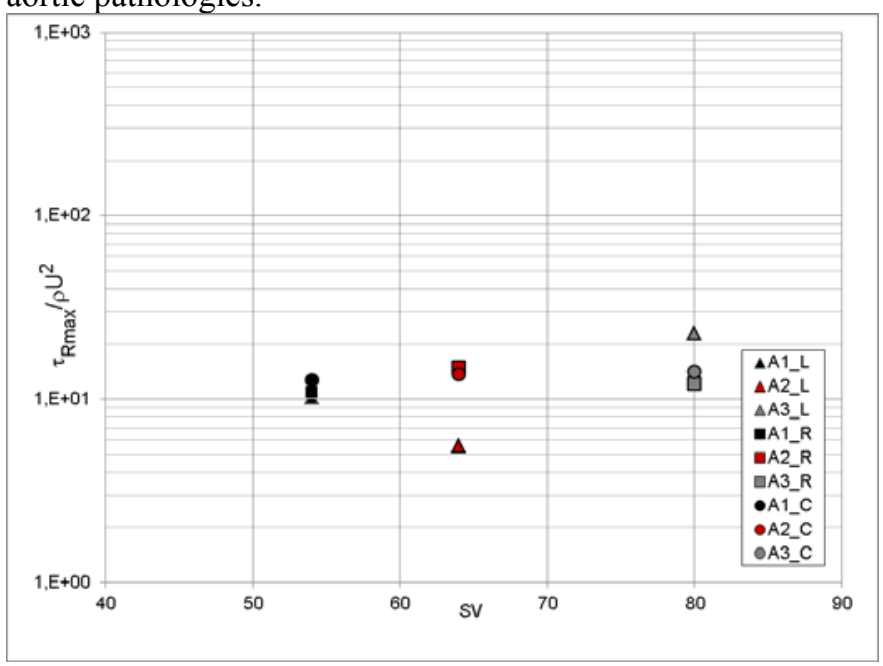

Figure 13. Maximum values of Reynolds shear stresses recovered in the regions $\mathrm{L}, \mathrm{C}$ and $\mathrm{R}$ for all the experiments performed.

\section{Acknowledgments}

This work was partially funded by the Ministero dell'Istruzione e della Ricerca Scientifica, PRIN 2009, Project No. 2009J7BL32.

\section{References}

[1] D.A Steinman, Image-based computational fluid dynamics modeling in realistic arterial geometries, Ann. Biomed. Eng. 30 (2002), pp. 483-497.

[2] D.M Wootton and D.N. Ku, Fluid Mechanics of Vascular Systems, Diseases, and Thrombosis, Annu. Rev. Biomed. Eng. (1999). pp. 299-329.

[3] A. Kheradvar and G. Pedrizzetti, Vortex Formation in the Cardiovascular System, Springer-Verlag, London, 2012.

[4] P.D. Stein and H.N. Sabbah, Measured turbulence and its effects on thrombus formation, Circ. Res. 35 (1974), pp. 608-614.

[5] M.J. Garcia, P. Vandervoort, W.J. Stewart, B.W. Lytle, D.M. Cosgrove, J.D. Thomas, and B.P. Griffin, Mechanisms of hemolysis with mitral prosthetic regurgitation study using transesophageal echocardiography and fluid dynamic simulation, J. Am. Coll. Cardiol. 27 (1996), pp. 399-406.

[6] G. Ismeno, A. Renzulli, A. Carozza, M. De Feo, M. Iannuzzi, P. Sante, and M. Cotrufo, Intravascular hemolysis after mitral and aortic valve replacement with different types of mechanical prostheses, Int. J. Cardiol. 69 (1999), pp.179-183.

[7] Y. Alemu and D. Bluestein, Flow-induced platelet activation and damage accumulation in a mechanical 
heart valve: Numerical studies, Artif. Organs 31 (2007), pp. 677-688.

[8] R.M. Nerem and W.A. Seed, An in vivo study of aortic flow disturbances, Cardiovasc. Res. 6 (1972), pp. 1-14.

[9] H. Nygaard, P.K. Paulsen, J.M. Hasenkam, E.K. Pedersen, and P.E.J. Rovsing, Turbulent stresses downstream of three mechanical aortic valve prostheses in human beings, J. Thorac. Cardiov. Sur. 107 (1994), pp. $438-446$.

[10] P.D. Stein and H.N. Sabbah, Turbulent blood flow in the ascending aorta of humans with normal and diseased aortic valves, Circ. Res. 39 (1976), pp. 58-65.

[11] C.J. Elkins, M. Markl, A. Iyengar, R. Wicker, and J.K. Eaton, Full-field velocity and temperature measurements using magnetic resonance imaging in turbulent complex internal flows, Int. J. Heat Fluid Fl. 25 (2004), pp. 702710 .

[12] P. Dyverfeldt, R. Gårdhagen, A. Sigfridsson, M. Karlsson, and T. Ebbers, On MRI turbulence quantification, Magn. Reson. Imaging 27 (2009), pp. 913-922.

[13] A.F. Stalder, M.F. Russe, A. Frydrychowicz, A. Bock, J. Hennig, and M. Markl, Quantitative 2D and 3D phase contrast MRI: Optimized analysis of blood flow and vessel wall parameters, Magnet. Reson. Med. 60 (2008), pp. 1218-1231.

[14] P.J. Kilner, G.Z. Yang, R.H. Mohiaddin, D.N. Firmin, and D.B. Longmore, Helical and retrograde secondary flow patterns in the aortic arch studied by threedirectional magnetic resonance velocity mapping, Circulation 88 (1993), pp. 2235-2247.

[15] H.G. Bogren, M.H. Buonocore, and R.J. Valente, Four dimensional magnetic resonance velocity mapping of blood flow patterns in the aorta in patients with atherosclerotic coronary artery disease compared agematched normal subjects, J. Magn. Reson. Imaging 19 (2004), pp. 417-427.

[16] M.D. Hope, T.A. Hope, S.E.S. Crook, K.G. Ordovas, T.H. Urbania, M.T. Alley, and C.B. Higgins, 4D Flow CMR in Assessment of Valve-Related Ascending Aortic Disease, J Am. Coll. Cardiol. Mediacl Imaging 4 (2011), pp. 781-787.

[17] J. Peacock, T. Jones, C. Tock, and R. Lutz, The onset of turbulence in physiological pulsatile flow in a straight tube, Exp. Fluid. 24 (1998), pp. 1-9.

[18] S. Jin, J. Oshinski, and D.P. Giddens, Effects of Wall Motion and Compliance on Flow Patterns in the Ascending Aorta, J. Biomech. Eng. 125 (2003), pp. 347354.

[19] S. Svensson, R. Gardhagen, E. Heiberg, T. Ebbers, D. Loyd, T. La"nne, and M. Karlsson, Feasibility of Patient Specific Aortic Blood Flow CFD Simulation, Lect. Notes Comput. Sci. 4190 (2006), pp. 257-263.

[20] K. Khanafer and R. Berguer, Fluid-Structure Interaction Analysis of Turbulent Pulsatile Flow Within a Layered Aortic Wall as Related to Aortic Dissection, J. Biomech. 42 (2009), pp. 2642-2648.

[21] A.C. Benim, A. Nahavandi, A. Assmann, D. Schubert, P. Feindt, and S.H. Suh, Simulation of Blood Flow in Human Aorta With Emphasis on Outlet Boundary
Conditions, Appl. Math. Model. 35 (2011), pp. 3175 3188.

[22] T. Yamaguchi, S. Kikkawa, and K. Parker, Simulation of nonstationary spectral analysis of turbulence in the aorta using a modified autoregressive or maximum entropy (ar/me) method, Med. Biol. Eng. Comput. 25 (1987), pp. 533-542.

[23] F. Menter, R. Langtry, and S. Volker, Transition Modelling for General Purpose CFD Codes Flow, Turbul. Combust. 77 (2006), pp. 277-303.

[24] U. Gulan, B. Luthi, M. Holzner, A. Liberzon, A. Tsinober, and W. Kinzelbach, Experimental study of aortic flow in the ascending aorta via Particle Tracking Velocimetry, Exp. Fluid 53 (2012), pp. 1469-1485.

[25] S. Fortini, G. Querzoli, S. Espa, and A. Cenedese, Threedimensional structure of the flow inside the left ventricle of the human heart, Exp. Fluids, 54 (2013), pp. 1-9.

[26] S. Espa, M.G. Badas, S. Fortini, G. Querzoli, and A. Cenedese, A Lagrangian Investigation of the flow inside the left ventricle, Eur. J. Mech. B-Fluid. 35 (2012), pp. 9-19.

[27] M. Vukicevic, S. Fortini, G. Querzoli, S. Espa, and G. Pedrizzetti, Experimental study of the asymmetric heart valve prototype, Eur. J. Mech. B-Fluid. 35 (2012), pp. 54-60.

[28] G. Querzoli, S. Fortini, and A. Cenedese, Effect of the prosthetic mitral valve on vortex dynamics and turbulence on the left ventricular flow, Phys. Fluids 22 (2010), pp. 041901-0419010.

[29] A. Cenedese, Z. Del Prete, M. Miozzi, and G. Querzoli, A laboratory investigation of the flow in the left ventricle of a human heart with prosthetic, tilting disk valves, Exp. Fluids 39 (2005), pp. 322-335.

[30] M. Grigioni, C. Daniele, V. D'Avenio, and V. Barbaro, Evaluation of the surface-averaged load exerted on a blood element by the Reynolds shear stress field provided by artificial cardiovascular devices, J. Biomech. 35 (2002), pp. 1613-1622.

[31] A.P. Yoganathan, J.D. Lemmon, and J.T. Ellis, Heart Valve Dynamics, in Biomechanics: Principles and Applications, Taylor \& Francis Group, LLC, 2003, pp. 189-203.

[32] R.N. Isnard, B.M Pannier, S. Laurent, G.M. London, and M.E. Safar, Pulsatile diameter and elastic modulus of the aortic arch in essential hypertension: a noninvasive study, J Am Coll Cardiol. 13 (1989), pp. 399-405.

[33] D. Baumgartner, C. Baumgartner, G. Mátyás, B. Steinmann, J. Löffler-Ragg, E. Schermer, U. Schweigmann, I. Baldissera, B. Frischhut, J. Hess, and I. Hammerer, Diagnostic power of aortic elastic properties in young patients with Marfan syndrome. J. Thorac. Cardiovasc. Surg. 129 (2005), pp. 730-739.

[34] E.A. Cowen and S.G. Monismith, A hybrid digital particle tracking velocimetry technique, Exp. Fluids 22 (1997), pp. 199-211.

[35] B.D Lucas and T. Kanade, An iterative image registration technique with an application to stereo vision, in: Proceedings of the 1981 DARPA imaging understanding workshop, Washington, DC (1981), pp. 121-130. 
[36] C. Tomasi and T. Kanade, Shape and motion from image streams: a factorization method-part 3. Detection and tracking of point features, Technical report CMU-CS Carnegie Mellon University, Pittsburgh, Pennsylvania (1991), pp. 91-132.

[37] A.A. Fontaine, J.T. Ellis, T.M. Healy, J. Hopmeyer, and A. P. Yoganathan, Identification of peak stresses in cardiac prostheses. A comparison of two-dimensional versus three-dimensional principal stresses analyses, ASAIO J. 42 (1996), pp. 154-163.
[38] P.L. Blackshear, Mechanical haemolysis in flowing blood. In: Fung YC (ed) Biomechanics (1972) Its foundation and objectives. Prentice Hall, Englewood Cliffs, New Jersey.

[39] W.L. Lim, Y.T. Chew, T.C. Chew, and H.T. Low Pulsatile flow studies of a porcine bioprosthetic aortic valve in vitro: PIV measurements and shear-induced blood damage, J. Biomech. 34 (2001), pp. 1417-1427.

[40] G. Querzoli, S. Fortini, S. Espa, M. Costantini, and F. Sorgini, Fluid dynamics of aortic root dilation in Marfan syndrome, J. Biomech. 47 (2014), pp. 3120-3128. 